\title{
Cardiac Disorder Classification by Electrocardiogram Sensing Using Deep Neural Network
}

\author{
Ali Haider Khan (D), ${ }^{1}$ Muzammil Hussain $\mathbb{D}^{1},{ }^{1}$ and Muhammad Kamran Malik ${ }^{2}$ \\ ${ }^{1}$ Department of Computer Science, School of Systems \& Technology, University of Management and Technology, \\ Lahore 54000, Pakistan \\ ${ }^{2}$ Punjab University College of Information Technology, University of the Punjab, Lahore 54000, Pakistan \\ Correspondence should be addressed to Ali Haider Khan; s2018288001@umt.edu.pk
}

Received 28 January 2021; Revised 6 March 2021; Accepted 10 March 2021; Published 23 March 2021

Academic Editor: Atif Khan

Copyright (c) 2021 Ali Haider Khan et al. This is an open access article distributed under the Creative Commons Attribution License, which permits unrestricted use, distribution, and reproduction in any medium, provided the original work is properly cited.

\begin{abstract}
Cardiac disease is the leading cause of death worldwide. Cardiovascular diseases can be prevented if an effective diagnostic is made at the initial stages. The ECG test is referred to as the diagnostic assistant tool for screening of cardiac disorder. The research purposes of a cardiac disorder detection system from 12-lead-based ECG Images. The healthcare institutes used various ECG equipment that present results in nonuniform formats of ECG images. The research study proposes a generalized methodology to process all formats of ECG. Single Shoot Detection (SSD) MobileNet v2-based Deep Neural Network architecture was used to detect cardiovascular disease detection. The study focused on detecting the four major cardiac abnormalities (i.e., myocardial infarction, abnormal heartbeat, previous history of MI, and normal class) with $98 \%$ accuracy results were calculated. The work is relatively rare based on their dataset; a collection of 11,148 standard 12-lead-based ECG images used in this study were manually collected from health care institutes and annotated by the domain experts. The study achieved high accuracy results to differentiate and detect four major cardiac abnormalities. Several cardiologists manually verified the proposed system's accuracy result and recommended that the proposed system can be used to screen for a cardiac disorder.
\end{abstract}

\section{Introduction}

According to the Centers for Disease Control and Prevention (CDC) and the American Health Monitoring Organization, the leading cause of death is cardiovascular disease [1]. CDC revealed that $74 \%$ of the population is affected yearly by heart disease. Cardiovascular diseases can be prevented if an effective diagnostic is made at the initial stages [2]. Modern medical science has shown substantial and potent solutions to cope with heart-related problems. In the era of technology, medical issues can be tackled with advanced techniques of Information technology. The most common heart disease detection technique is based on electrocardiogram (ECG), angiography screening, and blood test. The ECG test is also referred to as the diagnostic assistant tool for screening heart diseases [3]. ECG is a visual signal captured or measured by placing electrodes on the body's surface to detect voltage changes (Figure 1).
ECG represents the possible cardiac abnormalities in their ST segments: normally, the rises in ST segments, changes in segments, or flipping of $T$ waves, or new $Q$ wave; these abnormal segments reflect cardiac disease symptoms.

Researchers [4-6] investigated many techniques for automatically detecting cardiovascular disease using machine and deep learning techniques, typically by using ECG in one or twodimensional voltage amplitude data represented as time-series signals. To classify ECG signals in time series, different methods are explored and show a substantial result. Ubeyli [7] used an eigenvector method for feature extraction and to classify ECG beats. Sharma et al. [8] used time frequency-based ECG signals for feature extraction of the eigenvalue decomposition of Hankel matrix and Hilbert transform and used the random forest to detect the cardiovascular disorder.

In the current era, systems are being developed for the automatic detection of cardiac-related issues. These systems predict high accuracy results based on one-dimensional ECG 


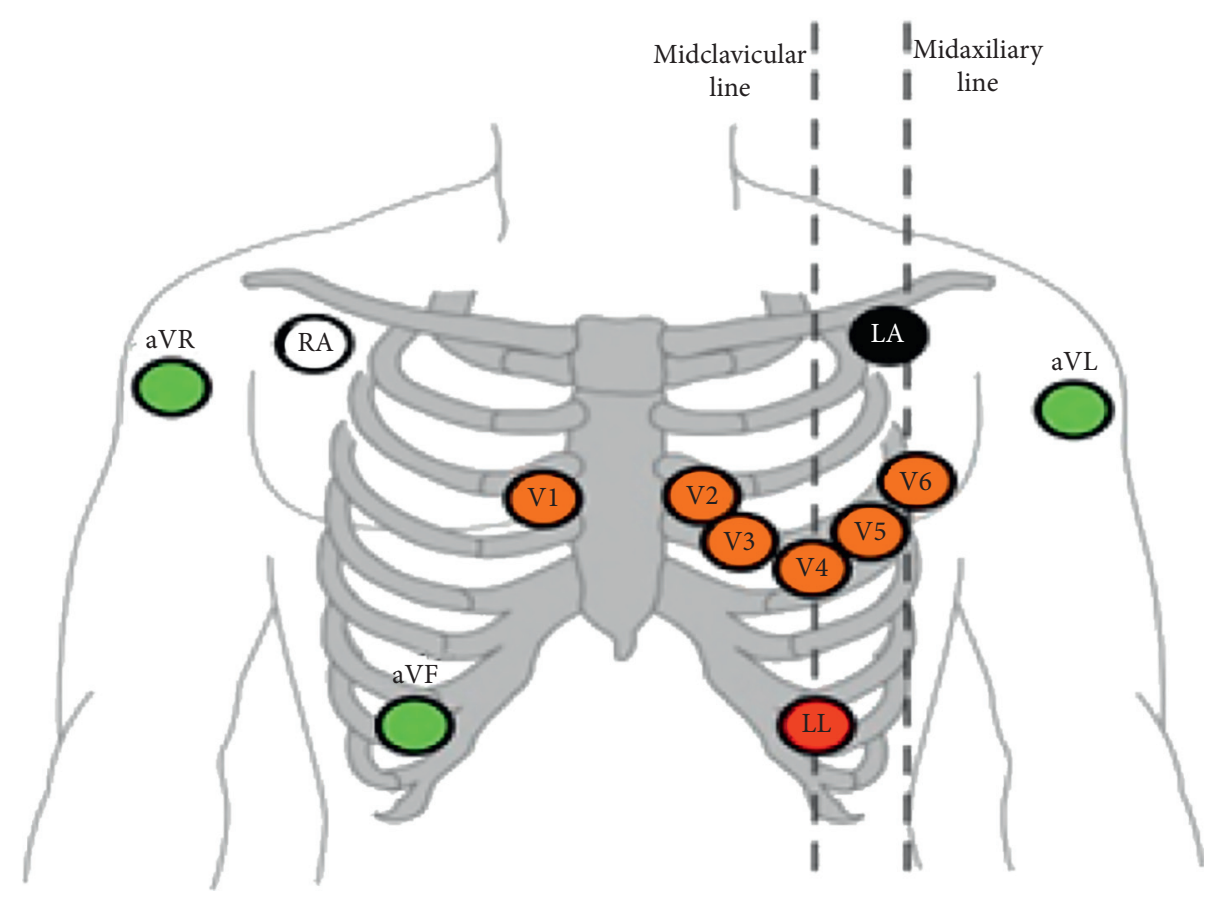

FIgUre 1: ECG electrodes.

beats signals but are still not adopted as tools in health care institutes. The main areas that affect the success of these approaches, i.e., selection of feature, extraction techniques, types of classification algorithms, and the most important, the use of imbalanced data for classification can reduce the recognition accuracy of the minority class [6]. Moreover, state-of-the-art studies represents the classical methods to show high accuracy in classification and detection tasks on one lead-based ECG images instead of standard 12-lead-based ECG images. Furthermore, the healthcare institutes used various ECG equipment, presenting results in nonuniform formats of ECG images. The state-of-the-art research was unable to propose a generalized methodology for nonuniform formats of ECG images. The availability of 12-lead-based ECG source images is not publicly available for researchers. In this research, the main focus is to provide a novel automatic detection tool relatively similar and adaptable for the cardiac hospitals to process the 12-lead-based ECG images.

Automated cardiac disorder detection via a deep neural network using 12-lead-based ECG image processing is critical. Deep neural network works in the same fashion as a human brain, founded on mathematical formulas/models. The mathematics of functional principles of deep neural networks targets to understand and recognize the pattern among different components. The deep neural network's fundamental unit is a neuron trained by repetitive tasks and gets experienced just like a human brain through acquired knowledge attained in training. The focus of training and acquiring knowledge is to establish a connection between input and output. After training, the system is capable of detecting the objects about what it has been trained.

The primary motivation of this study is to develop an efficient automated model for the diagnosis of cardiac disorder on ECG that can feasibly be implemented on portable healthcare devices. Figure 2 shows the structure of forward pass convolutional neural network with SSD detector.

The paper is organized into seven sections. The research community's related work in the current field is presented in section 2 with a close comparison. Section 3 illustrates the collection of the dataset used in this study, and data preprocessing techniques are discussed in section 4 . Section 5 illustrates the methodology with the proposed cluster. Simulated results are discussed in section 6 . The paper is concluded and discourses the future directions of our work in section 7 .

\section{Related Work}

In the past few decades, the research community has focused on artificial intelligence by working in digital image processing, computer vision, and machine learning to provide a platform between human and machine theory [6-9]. This work is widely recognized by several companies and medical fields for classification, detection, and identification of cardiac disorder, which play a vital role in the health community. Techniques used for the identification of cardiac disorder using deep learning have been focused on for many years.

In recent studies, the state-of-the-art research on deep learning gained the potential growth with satisfactory results in detection and classification tasks on medical images [9]. Most studies treated dimensional ECG signals as time-series classification by keeping in the view of deep learning. For example, PCGs and ECGs are used to diagnose heart disease. PCGs (also known as heart sound auscultation) is commonly 


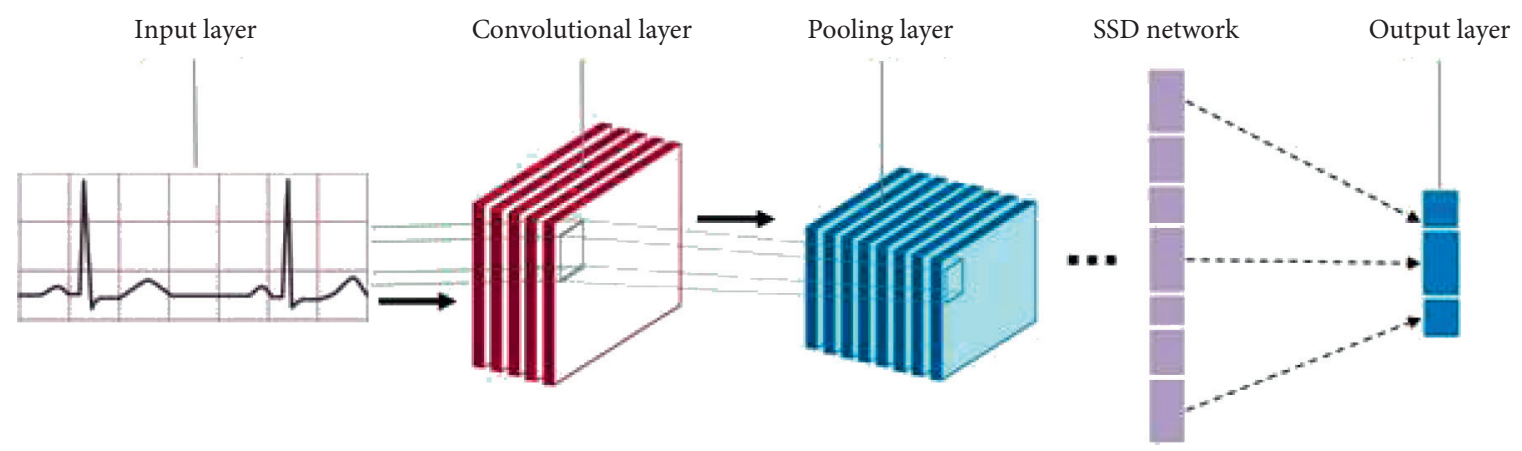

Figure 2: Structure of a forward pass convolutional neural network with SSD detector.

listened to or recorded by practitioners through a stethoscope, identifying the heart irregularities. For this reason, heart signals have been critically studied to make a diagnosis [10-13]. CNN is considered a state-of-the-art tool for detecting and classification heart signals and has been studied with several variations like 1-dimensional, 2-dimensional, or the combination of both [14, 15]. Similarly, Noman et al. [15] proposed a framework based on 1-dimensional CNN for direct feature learning from raw heart signals and 2-dimensional CNN, which takes 2-dimensional time-frequency feature maps. Xia et al. [16] proposed a model for automatic wearable ECG classification. Huang et al. [17] transformed five types of heartbeats' signals into time-frequency spectrograms and then trained a $2 \mathrm{D}-\mathrm{CNN}$ for classifying arrhythmia types. Lu et al. [18] transformed the $1 \mathrm{D}$ signals into $2 \mathrm{D}$ images by joining the dots of $1 \mathrm{D}$ signals. Ji et al. [19] also used a 1D signal, converted all signals into 2D, and used R-CNN for ECG classification.

In the state-of-the-art research studies, these time-series data used feature selection manually, which is not adaptable in different application environments. Moreover, ECG's time-series data with signal leads are not appropriate for stable baseline wanders, muscle contraction, and power line interface. In general, the practical methods normally adopted by a cardiologist for screening cardiac patients are 12-lead-based ECG images. In standard 12-lead-based ECG images, six leads are considered "limb leads" because they are placed on the individual's arms and/or legs. The other six leads are considered "precordial leads" because they are placed on the torso (precordium). The six limb leads are lead I, II, III, aVL, aVR, and aVF. The six precordial leads are called leads V1, V2, V3, V4, V5, and V6. Figure 3 shows sample 12-lead-based ECG image used in this study.

As concerned with ECG images, this research study is unique. In the real, collection of ECG images is done by recording or screening from Telehealth ECG diagnostic tool used in healthcare institutes. It is hard to gain these 12-leadbased on ECG images. Moreover, these images are used as a standard tool to detect the cardiac disorder in real-time applications.

In this research, the main focus is to provide a novel automatic detection tool relatively similar and adaptable for cardiac hospitals by using a deep neural network (DNN). The DNN has been widely recognized as a reliable approach to directly detect the characteristics of features

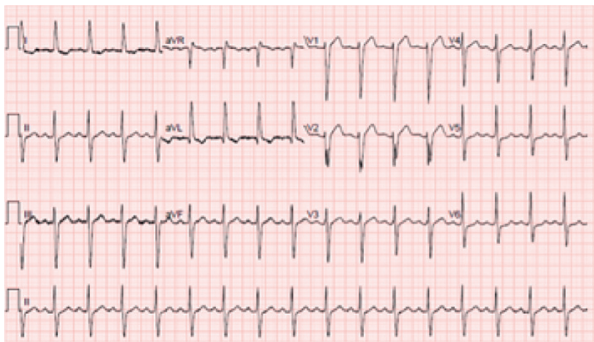

Figure 3: Sample ECG image.

from medical images [20, 21]. DNN is best suited for medical images problem; that is the main reasons authors used SSD MobileNet V2, a deep neural network-based architecture to detect the cardiac disorder on 12-lead-based ECG images.

\section{Dataset}

The steps involved in collecting the 12-lead-based ECG Images data required to develop the system is thoroughly described in this section.

3.1. Link Identification. Data resource identification was the main and primary challenge for the cardiac disease detection system. Consequently, in the first phase, various sites, repositories were explored. However, in spite of all efforts, all publicly available datasets that can be collected are timeseries data. Nevertheless, those available datasets are inconsistent with the data needed for this study. The manual collection of 12-lead-based standard ECG images directly from the cardiac institute is a difficult task. The authors approached one of the best cardiac institutes, i.e., Ch. Pervaiz Elahi Institute of Cardiology Multan, Pakistan, for data collection. The manual collection of ECG images from the cardiac institute took several months. The ECG images collected have been endorsed and annotated by numerous cardiologists and reexamined by the domain experts who have experience in ECG interpretation.

3.2. Data Description. Table 1 shows the total numbers of Images used for cardiac disorder detection used for each class. 
TABle 1: Data description.

\begin{tabular}{lccc}
\hline Sr. & Class & 12-lead ECG & Total images \\
\hline 1 & Myocardial infarction (MI) & 240 & 2880 \\
2 & Abnormal heartbeat & 233 & 2796 \\
3 & Previous history of MI & 172 & 2064 \\
4 & Normal & 284 & 3408 \\
\hline
\end{tabular}

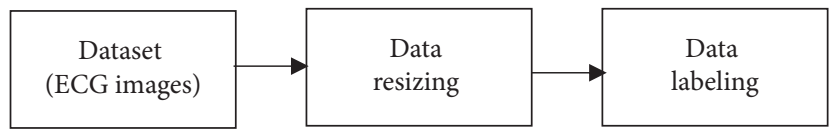

FIGURE 4: Data preprocessing.

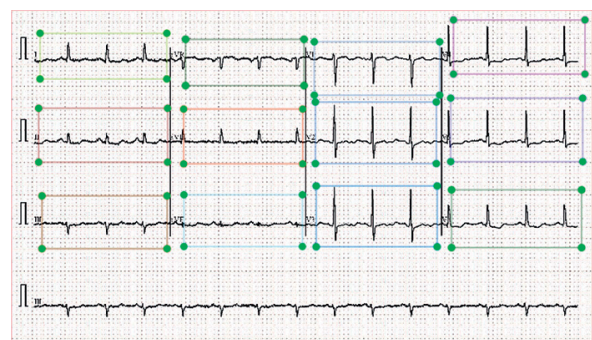

Abnormal heartbeat

(a)

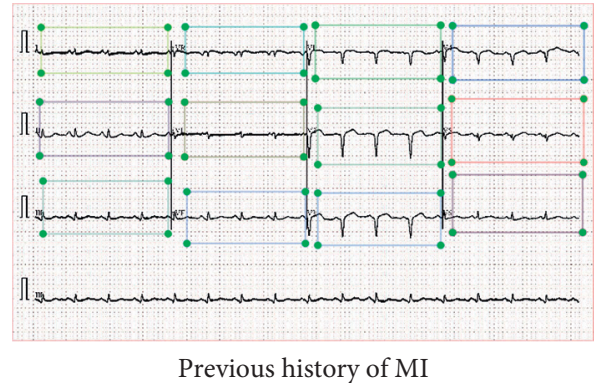

(c)

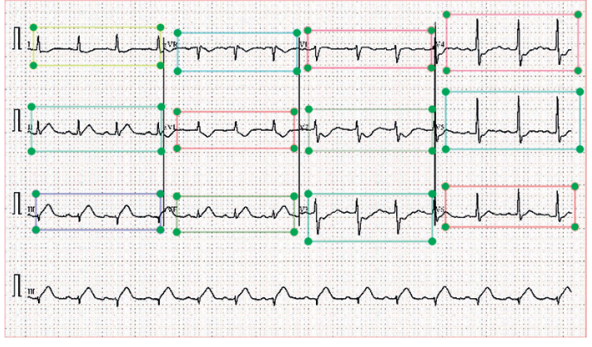

Myocardial infarction

(b)

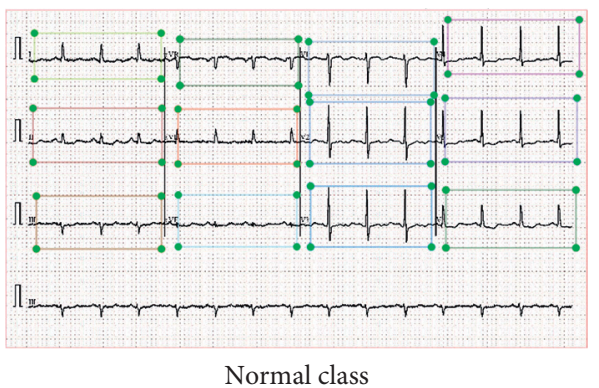

(d)

Figure 5: (a) Abnormal heartbeat; (b) myocardial infarction; (c) previous history of MI; (d) normal Class.

3.3. Data Availability. This study has collected data uploaded to a data repository [22], which contains complete data-related information to help the scientific community.

\section{Data Preprocessing}

Data preprocessing is an important step before inputting the data into the model for training. In this phase, data can be organized into meaningful information. Figure 4 shows the steps used in data preprocessing.

4.1. Data Resizing. The ECG images are higher than the $800 \mathrm{~KB}$ in size, requiring lots of time to train the algorithm, so all images required modification. The ECG images were modified to less than $300 \mathrm{~KB}$ in size to achieve more training steps in lesser time.
4.2. Data Labeling. This study collected data required to label all 12 leads of each ECG image before training. In this study, LabelImg tool is used to label the dataset classes, which means authors labelled 48 objects of all four classes shown in Figure 5 that our trained model will need to detect.

\section{Methodology}

The methodology used in this paper is to process the 12-leadbased ECG images to detect a cardiac abnormality. Single shot detector (SSD) MobileNet v2 is used in this study for model design. SSD is used to detect the objects that can classify and locate the objects in one step. The block diagram of the model used in this research study is shown in Figure 6.

5.1. Model Configuration. In this study, the model is implemented using Tensorflow API and Google Colab to 


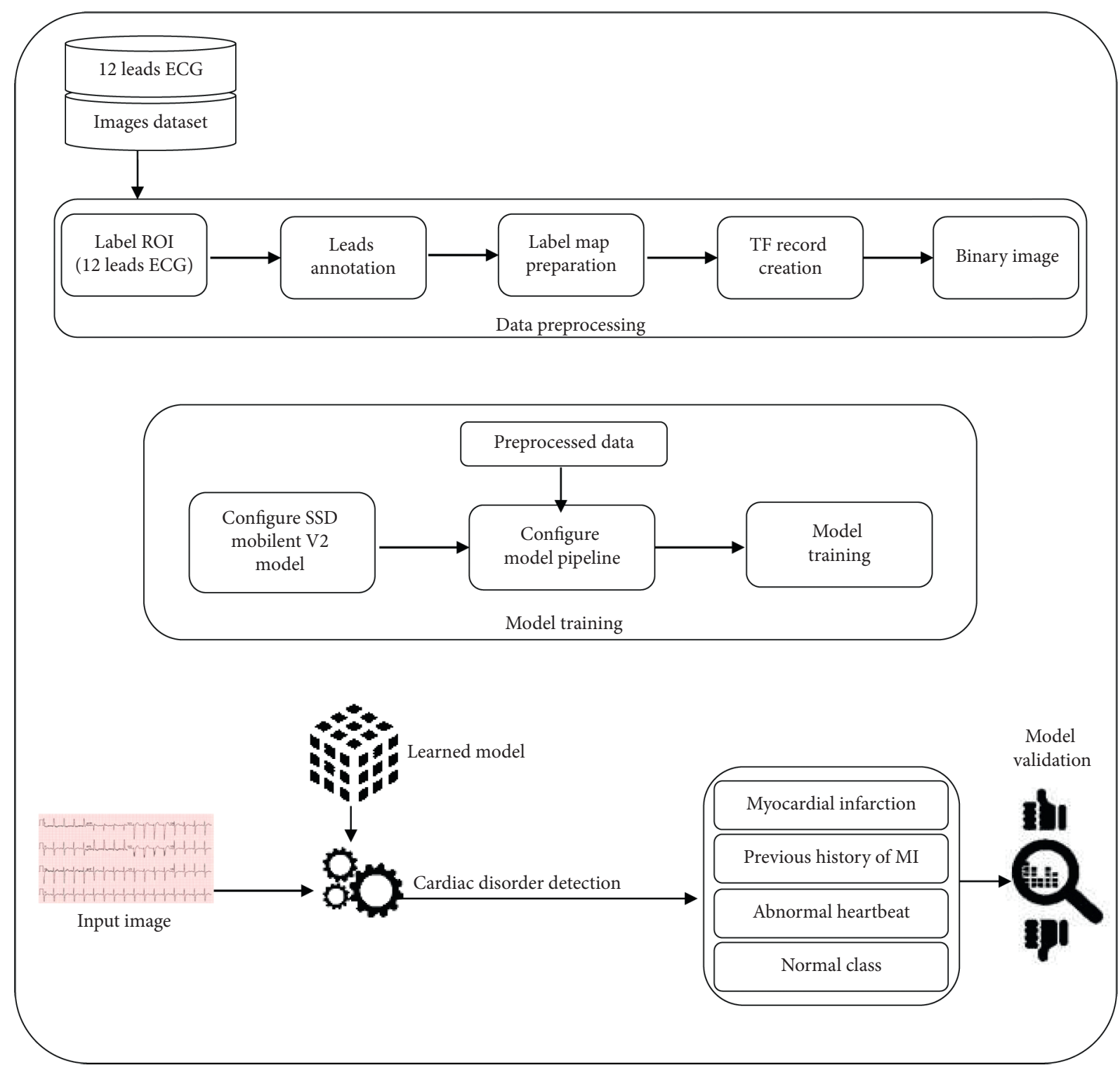

FIgURE 6: Research model.

build and train a cardiac disorder detector, using pretrained SSD MobileNet V2. In this study authors used the batch size of 24 and $200 \mathrm{~K}$ training steps for model training. Complete detail of the implementation with working source code is uploaded and can be seen on the GitHub of this project [23].

5.1.1. Dataset Splitting. The dataset used in this study is split so that $80 \%$ of the images are used for training the algorithms, while the remaining $20 \%$ is used for testing each class.

5.1.2. Number of Classes. Classes mean the number of objects that the model should learn and detect after training. In this case, the algorithms are responsible for detecting 4 classes (i.e., normal, abnormal heartbeat, myocardial infarction, and previous history of MI), and each class contains 12 leads; therefore, the number of objects is set to 48 .

5.1.3. Learning Rate. A default learning rate of 0.0002 has been used to train the algorithms.

5.2. Training. Once all hyperparameters are configured, then the training process starts with an average step speed of $0.50 \mathrm{~s}$. Training will take almost 4 days to complete the desired steps. The model will validate itself on the test dataset on each iteration and then display the prediction accuracy and loss. A sufficient number of steps are required for a better prediction of the model. Figure 7 shows the average loss (price paid for the wrong detection) of the model. 


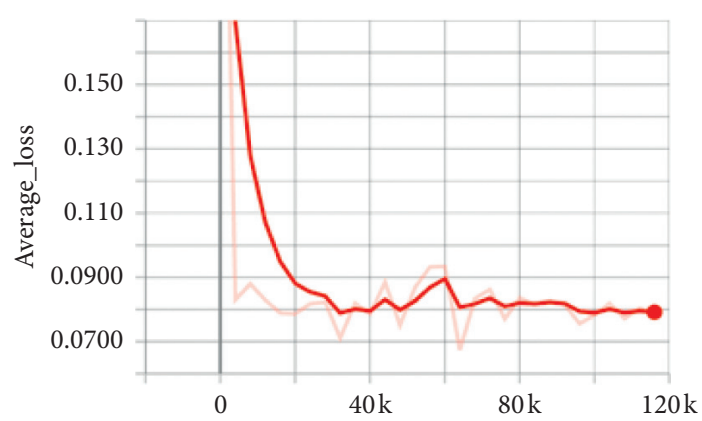

Figure 7: Average loss.

TABle 2: Model evaluation.

\begin{tabular}{lr}
\hline Class & Accuracy \\
\hline Myocardial infarction (MI) & 98.33 \\
Abnormal heartbeat & 97.22 \\
Previous history of MI & 98.28 \\
Normal & 96.18 \\
\hline
\end{tabular}
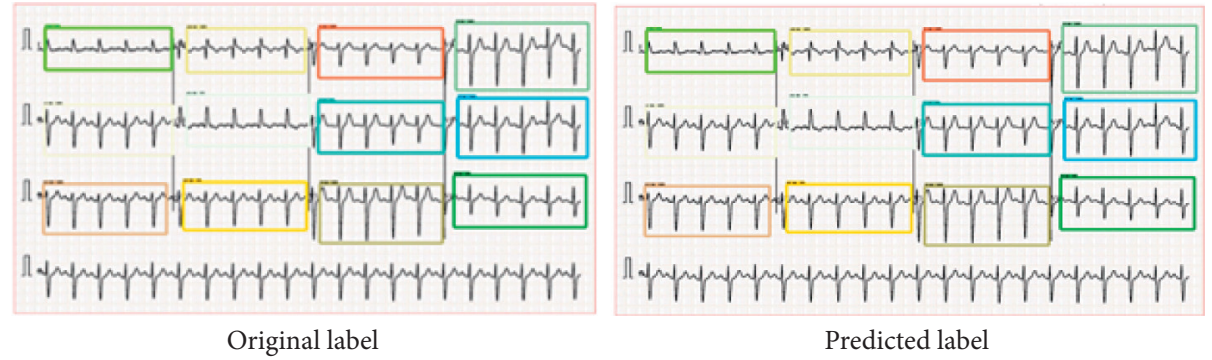

Figure 8: Predicted results.

5.3. Code Availability. The code used in this study can be found at https://github.com/alijiskani/ECG_Object_Detec tion, which contains code configuration files, source code, and a user manual.

\section{Results}

SSD MobileNet V2 was used to detect cardiovascular disease detection. High accuracy results were calculated for cardiac disease detection. The mean average precision against all four classes is evaluated. The study showed high accuracy results to differentiate and detect four cardiac abnormalities. "Myocardial Infarction" and "Previous History of MI" both classes achieved high accuracy among the results of all four classes. Table 2 shows the mean average precision (mAP) of all classes.

Figure 8 shows the original label and the predicted output results from the proposed model and their accuracy results.

The authors compare the cardiac disorder detection system performance with existing research studies related to
ECG cardiac classification works. However, the mentioned work has a different dataset based on time-series data and different types of classes, so it is not justified to directly compare their results. This study achieved remarkable results on the detection of four cardiac abnormalities and achieved high accuracy results. Table 3 shows the comparative results related to the study.

6.1. Confusion Matrix. The confusion matrix presents true positive, false positive, false negative, and true negative categories in each class (i.e., myocardial infarction, abnormal heartbeat, previous history of MI, and normal class). Table 4 displays the confusion matrix of the related study.

The class "myocardial infarction" generates the highest average score. The two major aspects of good prediction are low variance and large datasets. The training dataset has an important and effective role while learning high-level attributes, but the variance plays a vital role in emphasizing key features. 
TABLE 3: Result comparison.

\begin{tabular}{|c|c|c|c|c|c|}
\hline Classifier & Work & Data set & Publicly available & Accuracy & Total classes \\
\hline SSD MobileNet V2 & Proposed & 12-lead ECG images & Yes (now available) & 98.33 & 4 \\
\hline $\mathrm{CNN}$ & Acharya et al. [24] & Time series & No & 94.90 & 2 \\
\hline $1 \mathrm{D} \mathrm{CNN}$ & Kiranyaz et al. [25] & Time series & Yes & 94.73 & 2 \\
\hline $\mathrm{NN}$ & Inan et al. [26] & ECG beats & Yes & 95.20 & 2 \\
\hline Logistic regression & Sadhukhan et al. [27] & Multilead ECG & No & 95.60 & 2 \\
\hline $\mathrm{CNN}$ & Lodhi et al. [28] & 12-lead ECG & Yes & 93.53 & 2 \\
\hline
\end{tabular}

TABLe 4: Confusion matrix.

\begin{tabular}{|c|c|c|c|c|}
\hline & Myocardial infarction (MI) & Abnormal heartbeat & Previous history of MI & Normal \\
\hline Myocardial infarction (MI) & 96.22 & 1.89 & 1.89 & 0.00 \\
\hline Abnormal heartbeat & 3.70 & 93.59 & 1.35 & 1.36 \\
\hline Previous history of MI & 0.00 & 1.61 & 96.78 & 1.61 \\
\hline Normal & 0.00 & 2.75 & 3.51 & 93.74 \\
\hline
\end{tabular}

\section{Conclusion}

Cardiac disorder detection plays a significant role in medical and health science. The study focuses on processing the ECG images to detect cardiac abnormalities. The deep neural network has proven its capabilities in various applications of image processing and computer vision. This paper critically discusses the related work and analysis, specifically on cardiac disease detection. The study proposes a generalized methodology to process all formats of ECG. Single shoot detection (SSD) MobileNet v2 based Deep Neural Network architecture was used to detect cardiovascular disease detection. The study presented high accuracy results in differentiating and detecting four major cardiac abnormalities and showed remarkable results with an average accuracy of $98 \%$. Several cardiologists manually verified the proposed system's accuracy result and recommended that the proposed system be used to screen for cardiac disorder. The work is relatively rare based on their dataset using standard 12-lead-based ECG images used by cardiac professors in health care institutes.

This work can be extended by training a larger dataset, particularly on more cardiac abnormalities, validating the recognition ratio using DNNs. The extraction of advanced features on ECG images with image acquisition, adaptive image enhancement, and various boundary detection algorithms on various cardiac-related issues can be detected with medical experts' help with new developing tools. The way of learning domain adaptation is another big task for researchers to do in the future.

\section{Practical Implication}

This study achieved high accuracy results in detecting cardiac abnormalities, and several cardiologists manually verified the accuracy result of the proposed system. Moreover, this model can be implemented in the "mobile cardiac unit" as a beta version where no or fewer cardiac facilities are available.

\section{Data Availability}

The (12 Leads ECG Images) data used to support the findings of this study have been deposited in the (Mendeley) repository https://data.mendeley.com/datasets/gwbz3fsgp8/2.

\section{Conflicts of Interest}

The authors declare that they have no conflicts of interest regarding the study.

\section{References}

[1] Centers for Disease Control and Prevention, Heart Disease Fats, Centers for Disease Control and Prevention, Atlanta, GA, USA, 2020, https://www.cdc.gov/heartdisease/facts.htm.

[2] World Health Organization, Cardiovascular Diseases, Who, Geneva, Switzerland, 2020, http://www.who.int/mediacentre/ factsheets/fs317/en/..

[3] L. Wang, H. Zhang, K. C. Wong, H. Liu, and P. Shi, "Physiological-model-constrained noninvasive reconstruction of volumetric myocardial transmembrane potentials," IEEE Transactions on Bio-Medical Engineering, vol. 57, no. 2, pp. 296-315, 2010.

[4] Q. Zhang, D. Zhou, and X. Zeng, "HeartID: a multiresolution convolutional neural network for ECG-based biometric human identification in smart health applications," IEEE Access, vol. 5, pp. 11805-11816, 2017.

[5] U. R. Acharya, S. L. Oh, Y. Hagiwara et al., "A deep convolutional neural network model to classify heartbeats," Computers in Biology and Medicine, vol. 89, pp. 389-396, 2017.

[6] C. Potes, S. Parvaneh, A. Rahman, and B. Conroy, "Ensemble of feature-based and deep learning-based classifiers for detection of abnormal heart sounds," in Proceedings of the 2016 Computing in Cardiology Conference (CinC), Vancouver, Canada, September 2016.

[7] E. D. Übeyli, "Combining recurrent neural networks with eigenvector methods for classification of ECG beats," Digital Signal Processing, vol. 19, no. 2, pp. 320-329, 2009.

[8] R. R. Sharma, M. Kumar, and R. B. Pachori, "Automated cad identification system using time frequency representation 
based on eigenvalue decomposition of ECG signals," Machine Intelligence and Signal Analysis, vol. 748, pp. 597-608, 2019.

[9] P. Hao, K. You, H. Feng et al., "Lung adenocarcinoma diagnosis in one stage," Neurocomputing, vol. 392, pp. 245-252, 2020.

[10] O. Deperlioglu, "Segmentation of heart sounds by Re-sampled signal energy method, BRAIN," Broad Research in Artificial Intelligence and Neuroscience, vol. 9, no. 2, 2018.

[11] S. Ali, S. M. Adnan, T. Nawaz, M. Obaid Ullah, and S. Aziz, "Human heart sounds classification using ensemble methods," Technical Journal, University of Engineering and Technology (UET), vol. 22, no. No. I, pp. 113-120, 2017.

[12] L. Bahekar, A. Mishal, M. Bisen, D. Koche, and S. Alone, "Heart valve diseases detection using anfis and wavelet transform," International Journal of Research in Science \& Engineering, vol. 3, no. 2, pp. 279-291, 2017.

[13] M. V. Shervegar and G. V. Bhat, "Automatic segmentation of Phonocardiogram using the occurrence of the cardiac events," Informatics in Medicine Unlocked, vol. 9, no. 1, pp. 6-10, 2017.

[14] B. Xiao, Y. Xu, X. Bi, J. Zhang, and X. Ma, "Heart sounds classification using a novel 1-D convolutional neural network with extremely low parameter consumption," Neurocomputing, vol. 392, pp. 153-159, 2020.

[15] F. Noman, C. M. Ting, S. H. Salleh, and H. Ombao, "Shortsegment heart sound classification using an ensemble of deep convolutional neural networks," in Proceedings of the ICASSP 2019-2019 IEEE International Conference on Acoustics, Speech and Signal Processing (ICASSP), pp. 1318-1322, Brighton, UK, May 2019.

[16] Y. Xia, Z. Gao, H. Zhang, H. Zhang, and S. Li, “An automatic cardiac arrhythmia classification system with wearable electrocardiogram," IEEE Access, vol. 6, no. 99, p. 1, 2018.

[17] J. Huang, B. Chen, B. Yao, and W. He, "ECG arrhythmia classification using stft-based spectrogram and convolutional neural network," IEEE Access, vol. 7, pp. 92871-92880, 2019.

[18] W. Lu, H. Hou, and J. Chu, "Feature fusion for imbalanced ecg data analysis," Biomedical Signal Processing and Control, vol. 41, pp. 152-160, 2018.

[19] Y. Ji, S. Zhang, and W. Xiao, "Electrocardiogram classification based on faster regions with convolutional neural network," Sensors, vol. 19, no. 11, p. 2558, 2019.

[20] G. Litjens, T. Kooi, B. E. Bejnordi et al., "A survey on deep learning in medical image analysis," Medical Image Analysis, vol. 42, pp. 60-88, 2017.

[21] C. Park, C. C. Took, and J.-K. Seong, "Machine learning in biomedical engineering," Biomedical Engineering Letters, vol. 8, no. 1, pp. 1-3, 2018.

[22] A. H. Khan and M. Hussain, "ECG images dataset of cardiac and COVID-19 patients," Mendeley Data, vol. 1, 2020.

[23] GitHub repository model code, ECG object detection, 2020, https://github.com/alijiskani/ECG_Object_Detection.

[24] U. R. Acharya, H. Fujita, O. S. Lih, Y. Hagiwara, J. H. Tan, and M. Adam, "Automated detection of arrhythmias using different intervals of tachycardia ECG segments with convolutional neural network," Information Sciences, vol. 405, pp. 81-90, Sep. 2017

[25] S. Kiranyaz, T. Ince, and M. Gabbouj, "Real-Time PatientSpecific ECG Classification by 1-D Convolutional Neural Networksfic ECG classiffication by 1-D convolutional neural networks," IEEE Transactions on Biomedical Engineering, vol. 63, no. 3, pp. 664-675, 2016.

[26] O. T. Inan, L. Giovangrandi, and G. T. A. Kovacs, "Robust neural-network-based classification of premature ventricular contractions using wavelet transform and timing interval features," IEEE Transactions on Biomedical Engineering, vol. 53, no. 12, pp. 2507-2515, 2006.

[27] D. Sadhukhan, S. Pal, and M. Mitra, "Automated Identification of Myocardial infarction using harmonic phase distribution pattern of ECG data," IEEE Transactions on Instrumentation and Measurement, vol. 67, no. 99, pp. 1-11, 2018.

[28] A. M. Lodhi, A. N. Qureshi, U. Sharif, and Z. Ashiq, "A novel approach using voting from ECG leads to detect myocardial infarction," in Proceedings of the SAI Intelligent Systems Conference, pp. 337-352, London, UK, September 2018. 